\title{
Genome-wide association analysis reveal the genetic reasons affect melanin spot accumulation in beak skin of ducks
}

Hehe Liu

Sichuan Agricultural University

Jianmei Wang

Sichuan Agricultural University

Jian Hu

Chinese Academy of Agricultural Sciences

Lei Wang

Sichuan Agricultural University

Zhanbao Guo

Chinese Academy of Agricultural Sciences

Wenlei Fan

Chinese Academy of Agricultural Sciences

Yaxi Xu

Chinese Academy of Agricultural Sciences

Dapeng Liu

Chinese Academy of Agricultural Sciences

Yunsheng Zhang

Chinese Academy of Agricultural Sciences

Ming Xie

Chinese Academy of Agricultural Sciences

Jing Tang

Chinese Academy of Agricultural Sciences

Wei Huang

Chinese Academy of Agricultural Sciences

Qi Zhang

Chinese Academy of Agricultural Sciences

Zhengkui Zhou

Chinese Academy of Agricultural Sciences

Shuisheng Hou ( $\nabla$ houss@263.net)

Chinese Academy of Agricultural Sciences 


\section{Research Article}

Keywords: skin melanin spot, duck, GWAS, genetic

Posted Date: June 25th, 2021

DOl: https://doi.org/10.21203/rs.3.rs-608516/v1

License: (c) (i) This work is licensed under a Creative Commons Attribution 4.0 International License. Read Full License

Version of Record: A version of this preprint was published at BMC Genomics on March 26th, 2022. See the published version at https://doi.org/10.1186/s12864-022-08444-5. 


\section{Abstract \\ Background}

Skin pigmentation is a broadly appearing phenomenon of most animals and humans in nature. Here we used a bird model to investigate why melanin spot deposits on the skin.

\section{Results}

We result shown that melanin deposition in bird skin was induced by growth age and ultraviolet UV radiation and determined by genetic factors. GWAS helped us to identify two major loci affecting melanin deposition, located on chromosomes 13 and 25, respectively. Fine mapping works narrowed the candidate regions to $0.98 \mathrm{Mb}$ and $1.0 \mathrm{Mb}$ on chromosome 13 and 25, respectively. The MITF and POU2F3 may be the causative genes and synergistically affect melanin deposition during duck skin. Furthermore, our data strongly demonstrated that the pathway of melanin metabolism contributes to melanin deposition on the skin.

\section{Conclusions}

We demonstrated that age and UV radiation induce melanin deposition in bird skin, while heredity is the fundamental factor. The MITF and POU2F3 likely played a synergistic effect on the regulation of melanin synthesis, and their mutations contribute to phenotypic differences in beak melanin deposition among individuals. It is pointed out that melanin deposition in the skin is related to the pathway of melanin metabolism, which provided insights into the molecular regulatory mechanisms and the genetic improvement of the melanin deposition in duck beak.

\section{Introduction}

Skin pigmentation is a broadly appearing phenomenon in most animals and humans. It is related to the health conditions and the age status. In humans, changes in skin color are often associated with age ${ }^{[1]}$. Although numerous efforts have been made to retard the skin aging process, the skin structure and function declined over time and long-term exposure to ultraviolet rays (UV) irradiation from the sunlight ${ }^{[2]}$. Eventually, the skin pigmentation of sun-exposed areas, i.e., face, neck, forearms, or dorsal surface of hands, becomes asymmetrical with age due to continuous sun exposure, and this mottled pigmentation develops a sign of aged $\operatorname{skin}^{[3,4]}$. However, in sunshade areas of skin, the pigmentation and reactive tanning decrease with age ${ }^{[5,6]}$. In birds, skin pigmentation increases with age also, e.g., the female Pekin duck accumulates a large amount of melanin in the beak skin during the breeding period. In poultry production, excessive melanin deposition in the skin directly affects the sensory quality and the desire of consumers to purchase. 
The colors of feathers, furs, and skin are mainly determined by melanocytes, which taking part in the synthesis of melanin pigments that play an essential role in cosmetic variation, heat regulation, and camouflage and simultaneously give protection against UV radiations ${ }^{[7]}$. Moreover, the accumulation of melanin pigments causes skin pigmentation ${ }^{[8]}$. In the young adult epidermis, melanocytes are pretty evenly distributed on the body at the rate of $1500 / \mathrm{mm}^{2}$ density ${ }^{[9,10]}$. However, after 30 years of age, the aging process is tied to a 10 to 20 percent per decade decrease in melanin production by melanocytes ${ }^{[11]}$. As a result, in aging skin, the asymmetrical distribution of melanocytes caused the formation of melanin spots under constantly sun-exposed.

Skin pigment deposition is also highly inherited and regulated by genetic factors ${ }^{[12]}$. The a-MSH is one of the significant inherited factors and acts mainly as an agonist of MC1R. The high incidence of MC1R mutations in individuals with red hair and light skin may be responsible for the reduced response to a$\mathrm{MSH}$, resulting in decreased melanin production and pigmentation caused by UV exposure ${ }^{[13]}$. Furthermore, it was shown that the polymorphism of MC1R is associated with ethnic differences in structural pigmentation and different responses to UV irradiation $[14,15]$.

Birds live most of the time outdoors and are more easily affected by ultraviolet radiation. Therefore, melanin stand out in hair follicles, skin, eyes, feathers, and scales ${ }^{[16]}$. Pekin duck is a famous whitefeathered duck breed globally, and the white feather is caused by genetic albinism ${ }^{[17]}$, which means very little melanin distributed in feathers and skin. The melanin spots on beak skin have been observed during the breeding period of female Pekin ducks. However, the underlying reason is still unclear. Here, we used duck as a model to investigate why melanin spots were deposited on the skin. The potential influencing factors, including age, sunlight exposure, and reproduction abilities, were estimated in ducks.

Furthermore, based on a collection of $223 \mathrm{~F}_{2}$ ducks crossed by Pekin ducks and mallards, we also performed a genome-wide association study (GWAS) to gain insights into the genetic factors influencing melanin deposition in the skin. These works provide insights into the molecular regulatory mechanisms and the genetic improvement of the melanin deposition in duck beak.

\section{Results And Analysis}

\section{Descriptive statistics of phenotypic traits}

We used the IPP 6.0 software to open the images and utilized its function to evaluate the amount of melanin spot and area of the duck beak (Fig. 1A). The melanin spot was mainly accumulated on the beak at the lateral growth period of ducks, and the amount of melanin spot increased gradually with age (Fig. 1B). However, the amount of melanin spot on the duck's beak exhibited an individual difference. The correlations between melanin deposition in beak skin and egg production, egg weight, feed intake, and duck feed-egg ratio were analyzed to test the impacts of the reproduction process on melanin spot accumulation. The results showed that all correlations were less than 0.2 (Fig. 1C), indicating only slight relations between them. Whereas the melanin spot of ducks feeding under an enclosed house and a 
semi-open breeding house was compared, it was observed that the former ones had fewer melanin spot than the latter ones (Fig. 1D), suggesting the sunlight exposure may be the precise cause of melanin spot in duck beak skin. The phenotypic data of melanin spot in beak skin, beak area, and melanin deposition per unit beak area of 223 female individuals were collected. All these phenotypes' values tend to a normal distribution (Fig. 1E).

\section{Genome-wide association analysis (GWAS)}

We performed GWAS based on the genotypes and phenotypes, and the Manhattan and Quantile-Quantile (QQ) plots are shown in Fig. 2. For MSA, a single association peak was observed on chromosome 2 ( $P=$ $2.76 \times 10^{-10}$; Fig. $\left.2 \mathrm{~A}\right), 13\left(P=2.5 \times 10^{-10}\right.$; Fig. $\left.2 \mathrm{~A}\right)$, and $25\left(P=7.59 \times 10^{-10}\right.$; Fig. $\left.2 \mathrm{~A}\right)$, respectively. For MSPBA, a single association peak was observed on chromosome $13\left(P=4.88 \times 10^{-11} ;\right.$ Fig. $\left.2 \mathrm{C}\right)$ and $25(P$ $=1.04 \times 10^{-13}$; Fig. $\left.2 \mathrm{C}\right)$, respectively. However, for the trait of BA, non-significant SNPs were observed (Fig. 2B). The QQ plots for MSA and MSPBA revealed that SNPs deviated from the distribution under the null hypothesis, which indicated a strong association between SNPs and phenotypes (Fig. 2). Therefore, the GWAS signals on chromosome 13 and 25 for MSA were overlapped with the signals associated with MSPBA. As the signals of the later ones were stronger than the former ones, we thus continually focused on the GWAS signals of MSPBA. For this trait, 46 SNPs on chromosome 13 and 25 SNPs on chromosome 25 reached the Bonferroni corrected significance threshold ( $\log _{10} P=8.16$, Table 11 and Table S2). These significant SNPs contributed a candidate genomic region ranged from 4.89 to $5.92 \mathrm{Mb}$ on chromosome 13 , and a region ranged from 3.35 to $4.47 \mathrm{Mb}$ on chromosome 25, respectively (Fig. 2). For the GWAS signal for the MSA trait, 12 significant SNPs that reached the Bonferroni corrected significance threshold $\left(-\log _{10} P=8.16\right)$ contributed a candidate genomic region ranging from 15.2 to $24 \mathrm{Mb}$ on chromosome 2 .

\section{Fine mapping the causative genomic region}

We conducted conditional GWAS of MSPBA using the loci in the candidate region by fitting the most significant SNP as a covariate factor (Fig. 3A\&B). It was observed that the association peaks for these two signals have vanished. No more SNPs reached the significant threshold line of $-\log _{10} P=8.16$. The results implied high linkage relationships for these SNPs. For the signal on chromosome 13, the regional plots showed 27 significant SNPs have a higher pairwise LD that reached an $R^{2} \geq 0.4$ with the leader significant SNP (Fig. 3C, Table S1). These SNPs in strongly pairwise LD supported a fine mapped region of $0.98 \mathrm{Mb}$ ranging from 4.95 to $5.93 \mathrm{Mb}$ on chromosome 13. For the signal on chromosome 25 , the regional plots showed 11 significant SNPs have a higher pairwise LD that reached an $R^{2} \geq 0.4$ with the leader significant SNP (Fig. 3D, Table S2). All these significant SNPs were identified as being located in a $\sim 1.0 \mathrm{Mb}$ region spanning from 3.45 to $4.47 \mathrm{Mb}$ on chromosome 25 because of the strong genetic correlations.

\section{Genes association with melanin spot deposition}


The fine-mapped region on chromosome 13 harbored 14 genes (Fig. 4A), which may play potential roles as candidate genes for the formation of melanin spots, including LOC101799721, LOC101799904, MITF, FRMD4B, LMOD3, ARL6IP5, UBA3, TMF1, EOGT, FAM19A4, LOC106014426, FAM19A1, LOC106014427, and SUCLG2. Among the 46 significant SNPs associated with MSPBA, 17 SNPs were distributed in the intron regions of the MITF gene. The leader significant SNP (Chr13: 5,019,135) in GWAS was located on the intron region of the MITF gene. Moreover, 7 SNPs located in the intron regions of the MITF have a higher pairwise LD that reached an $\mathrm{R}^{2} \geq 0.7$ with the leader significant SNP (Table S1). According to the global gene expression dataset based on the transcriptome, 10 out of 14 candidate genes were detected to be expressed in duck beak skin. Among them, only MITF and LOC106014426 have a relatively higher expression level in beak skin than in other tissues (Figure S1). The MITF was reported to play roles in melanocyte migration and melanin synthesis. Collectively, these data supported the MITF seemly as the causative gene for the formation of melanin spot in duck beak. The fine-mapped region on chromosome 25 harbored 42 candidate genes for the MSPBA trait. Surrounding the leader significant SNP (Chr25: 4286765) in GWAS, there are three genes, including POU2F3, ARHGEF12, and GRIK4 (Fig. 4B). According to the global gene expression dataset based on the transcriptome, 23 out of the 42 candidate genes expressed in duck beak skin with an average CPM value of more than 1 . Among the 23 candidate genes, 17 were annotated as functional genes, including LAYN, POU2AF1, HINFP, NLRX1, RNF26, C1QTNF5, MFRP, USP2, THY1, NECTIN1, TRIM29, OAF, POU2F3, and ARHGEF12 (Figure S2).

\section{A collaborate effects of MITF and POU transcription factors on melanin spot on duck beak skin}

We genotyped all 223 ducks using allele information of the two leader SNPs Chr13:5,019,135 and Chr25:42,867,656 and analyzed the impacts of genotypes by these two alleles on the phenotypes. The results demonstrated that these two loci had coordination effects on the MSPBA (Fig. 5A). These findings reminded us there might be regulatory relationships between the causative genes underlying the two GWAS signals, as some of the POU members were reported to play roles as transcription factors of the MITF gene. Therefore, we inferred there might be potential cis-regulatory relationships between the POU transcription factors underlying signal on chromosome 25 and the MITF gene underlying signal on chromosome 13. We thus downloaded the promoter sequence of the duck MITF gene, and the transcription factors and their binding sites were predicted by using online software PROMO. As a result, a total of $44 \mathrm{POU}$ transcription factors was predicted to be distributed within the promoter region of duck MITF. These POU transcription factors were included POU1F1A, POU1F1B, POU2F1, and POU2F2B (Fig. 5B, Table S3). Although the candidate genes POU2AF1 and POU2F3, underlying the GWAS signal on chromosome 25, were not included in the four predicted POU transcription factors, they shared higher sequence homology with other POU members, according to a molecular phylogenetic tree by using all duck POU gene family members (Fig. 5C). Subsequently, a gene expression cluster analysis of the candidate genes on chromosome 13 and 25 were performed using transcriptome data in beak skin of ducks at the ages of one day old and twenty weeks old. The cluster results demonstrated that POU2AF1, POU2F3, and MITF have a similar expression pattern. All of them increased at the stage of 20 weeks old (Fig. 5D), suggesting combined effects on melanin spot deposition on duck beak skin. 


\section{Discussion}

Melanin is produced from tyrosine catalyzed by tyrosinase undergone a series of catalytic reactions ${ }^{[18]}$. Age and sunlight exposure are the main causes of inducing the deposition of melanin spots on the human face and neck. In this study, we found that melanin deposition in duck beak skin increased with age. Meanwhile, the melanin deposition in the beak skin of ducks reared under the fully enclosed house was lighter than the ducks feed in the semi-open house system. Our results were consistent with the study by Maddodi et al. ${ }^{[19]}$ in humans, supporting that the melanin spot in the skin is related to age and UV irradiation for both birds and mammals.

In poultry production, it was considered that the deposition of melanin in duck beak skin might be linked to reproductive activities. Previous studies have demonstrated an estrogen receptor in normal human melanocytes ${ }^{[20,21]}$. Kim et al. ${ }^{[22]}$ found that estrogen can promote the transport of melanocytes to keratinocytes, resulting in the formation of chloasma. Moreover, estradiol at a definite concentration can also induce deeper skin pigmentation ${ }^{[23]}$. During the laying period of ducks, the melanin was deposited on the beak surface, and there were individual differences in the amount of melanin deposition. However, a low correlation was observed between melanin deposition in beak skin and reproduction abilities. Therefore, it suggested that the physiological metabolic processes did not cause the melanin deposition in duck beak skin during reproductive activities.

Based on GWAS analysis, three loci associated with melanin amount were identified in the duck genome, and these loci were located on chromosomes 2, 13, and 25, respectively. Our findings implied that melanin deposition in duck beak skin was affected by age and sunlight exposure and genetic factors. Similar results have also been reported in humans ${ }^{[12,24]}$. We believed that MITF and POU gene might be the essential candidate genes affecting melanin deposition in duck skin through further fine-mapping works and expression analysis. The MITF protein is considered to be a necessary regulator of melanocyte migration and differentiation ${ }^{[25]}$. Our findings implied that the melanin synthesis and metabolic pathway played a crucial role in inducing melanin deposition in the skin during age and sunlight exposure. Mutations in any genes related to melanin synthesis and metabolic pathway would affect melanin syntheses, such as MC1R, ENDRB, RAB27A, OA1, PMEL17, MLANA, GPNMB, Melastatin1, Aim1, TYR, TRP-1, TRP-2, and MART-1 ${ }^{[26]}$. Our findings in ducks also served as a reference for human beings to find the causative genes that affect melanin spots under UV irradiation.

Members of the POU transcription factor family can promote transcriptions of many genes related to development and metabolism ${ }^{[27]}$. This set of gene families shares a typical structure, the POU domain ${ }^{[28]}$. The name POU derives from the major transcription factors described in the family ${ }^{[27]}$. POU members have been declared to regulate the transcription of the MITF gene ${ }^{[28,29]}$. We found that there are 13 POU gene family members within the duck genome. A total of $44 \mathrm{POU}$ transcription factors were predicted to be distributed within the promoter region of the MITF, and they were POU1F1A, POU1F1B, POU2F1, and POU2F2B. In the candidate region of duck chromosome 25, we found two members of the POU gene 
family, namely POU2AF1 and POU2F3. The regulatory relationships between POU members and the MITF gene were also supported by the correlation analysis between allele frequencies and phenotypes, which suggested a coordination effect of these two loci on melanin deposition in duck beak skin. Collectively, our data indicated a collaboration effect of MITF and its POU transcription factors on melanin spot deposition on duck beak skin. However, their specific regulatory process needs to be further verified by more molecular biological evidence.

The variations in skin pigmentation are the results of adaptability to various geographical locations ${ }^{[24,30]}$. The pivotal function of pigmentation is the protection of the exposed skin against UV radiation ${ }^{[31]}$. Predictably, genetic variations within the genes involved in the pigmentation process caused phenotypic variations, such as skin pigmentation, hair color, eye color, freckling, and skin sensitivity to sunlight ${ }^{[32]}$. The ancestor of domestic ducks is the mallard, which is a kind of migratory bird, and the pigmentation variations in the skin are beneficial for adapting to a wide range of environmental conditions. We thus assumed that, after many mutations generated in the MITF and POU during the evolutionary process, the favorable mutations tended to be preserved in duck genomes, to increase the adaptability to different light environments. However, accompanying with age and constant UV irradiation, the asymmetrical distribution of melanocytes caused the formation of melanin spot in duck beak skin. In poultry production, some farmers believed that the melanin spot accumulation in beak skin of ducks is caused by fading of xanthophylls that cover the existing melanin. Nevertheless, our present study has not identified any genes, whose polymorphism related to xanthophylls synthesis were associated with the content of melanin in duck beak skins. The present study, laid new clues for understanding about genetic factors that can affect the melanin spot in the skin, however further investigation required to strengthen this hypothesis.

\section{Conclusion}

We demonstrated that age and UV radiation induce melanin deposition in bird skin, while heredity is the fundamental factor. The MITF and POU2F3 likely played a synergistic effect on the regulation of melanin synthesis, and their mutations contribute to phenotypic differences in beak melanin deposition among individuals. It is pointed out that melanin deposition in the skin is related to the pathway of melanin metabolism, which provide insights into the molecular regulatory mechanisms and the genetic improvement of the melanin deposition in duck beak.

\section{Materials And Methods}

\section{Animals}

Total $223 \mathrm{~F}_{2}$ female ducks were used in this study from reciprocal crosses of Mallards and Pekin ducks. The Mallards were obtained from the Aoji Duck Farm (hunting license available, Zhejiang, China). Pekin ducks were kept on Pekin Duck Breeding Center of the Chinese Academy of Agricultural Sciences (Pekin, China). For the orthogonal cross, 100 female Mallards and 10 male Pekin ducks were selected as parents. 
In the reciprocal cross, 4 male Mallards and 40 female Pekin ducks were selected as parents. For both cross-manner designs, one male duck was mated to 10 females. Thus, a total of 216 families was established using the $F_{1}$ hybrids. The cross works were constructed in 2015 , more than $2000 F_{2}$ ducks

were obtained for subsequent studies. Most of them were slaughtered for growth performance studies ${ }^{[17]}$. The left ducks were sequentially raised under the same conditions in individual cages for phenotype measurement in this current study.

\section{Genotyping}

Blood samples were collected from the wing vein of female ducks. A total of 223 blood samples were selected for DNA extraction using the phenol-chloroform protocol. The quality and quantity of DNA were examined by Nanodrop and agarose gel electrophoresis. The paired-end libraries were generated for each eligible sample using standard procedures. Besides, the average insert size was $500 \mathrm{bp}$, and the read length was $150 \mathrm{bp}$. All libraries were sequenced on an Illumina ${ }^{\circledR}$ Hiseq X-Ten platform in a Bio-company (Berry Genomics, Pekin, China) to average raw read sequence coverage of $5 \times$ for a paired end of $150 \mathrm{bp}$ (PE150).

The raw reads were filtered using NGS QC (v2.3.3) Toolkit with default parameters[33]. The clean reads were mapped to the duck reference genome (IASCAAS_PekingDuck_PBH1.5, GCF_003850225.1) with Burrows-Wheeler alignment (BWA aln)[34] using the default parameters. Next, SNPs calling was performed using GATK (version 3.5) exclusively ${ }^{[35]}$, and the output was further filtered using VCFtools (version 0.1 .15$)^{[36]}$. The SNPs were screened with the parameters of a minor allele frequency (MAF) > 0.05 , a max allele frequency $<0.99$, and the maximum missing rate was $<0.1$. All filtered SNPs were distributed on 29 autosomal chromosomes, Chr Z, Chr W, and Chr U (unplaced scaffolds) ${ }^{[17]}$.

\section{Phenotypes`collection}

Melanin spots in the beak skin of all ducks were photographed in the same conditions. First, the digital camera was set to a manual exposure manner, and each photo was based on identical exposure conditions, including exposure time and aperture. Then, the obtained images were imported into IPP 6.0 software (Media Cybernetics, USA), and afterward, they were magnified by the identical multiple. Next, using an irregular tool incorporated in the software, the area of interest (AOI) of each melanin spot was selected, and the geometric size of each region was measured. Finally, melanin spot area in duck beak skin (MSA) and beak area (BA) was measured, respectively, and the melanin distribution per unit area of beak skin (MSPBA) was calculated. Three replicates in each measurement and the average values were taken as the final phenotype.

To compare the melanin spots of duck beak skin among ages, 10 Pekin ducks were randomly selected at each age time point. Total 30 ducks at 60 -week-old were randomly chosen from fully enclosed house feeding (mainly with artificial light only instead of whole sunlight exposure during the growing period) and a semi-open breeding house (exposed to sunlight during the growing period), respectively, and melanin spots in beak skin of them were determined by the above-mentioned method. All other feeding 
management conditions were kept the same. The primary purpose was to compare the effect of sunlight on the deposition of melanin spots in beak skin. In addition, the reproduction abilities of $223 \mathrm{~F}_{2}$ female ducks were determined by egg production and feed conversion ratio.

\section{Whole-genome association analysis}

Population structure and cryptic relationships were considered to minimize false positives and increase statistical power. A mixed linear model program emmax was used for the association analysis ${ }^{[37]}$. The first three Principle component values (PCA eigenvectors) derived from whole-genome SNPs and the forward/backward cross were set as a fixed effect in the mixed model to correct for population stratification ${ }^{[38]}$. The random effect was the kinship matrix estimated among all individual whole-genome SNPs.

\section{Gene expression analyses}

Duck global expression dataset of mallards and Pekin ducks has been constructed previously ${ }^{[17]}$, where a global transcriptome project of ducks was conducted. First, the clean reads of RNA-seq paired-end reads were mapped against the Pekin duck reference genome (IASCAAS_PekingDuck_PBH1.5, GCF_003850225.1) using the Tophat read mapper. Then Htseq-count were runned for reads count per gene and calculate the CPM (counts per million) value of genes.

Genes that shared similar functions or have regulatory relations usually exhibit identical expression patterns. According to a classic cluster method, to demonstrate the potential regulatory effects of the predicted transcription factors on MITF transcriptions, their expression levels based on transcriptome data of 18 individuals were collected for gene expression clusters, according to a classic cluster method $^{[39]}$. These 18-transcriptome data were performed in beak skin of the ducks at ages of 1 day old $(n=12)$ and 20 weeks old $(n=6)$.

\section{Transcription factors' prediction}

The online software PROMO (http://alggen.Isi.upc.es/cgi-bin/promo_v3/promo/promoinit.cgi? dirDB=TF_8.3) was used for predicting transcription factors and their binding sites on the cis-regulatory region of the duck MITF gene. The $0.5 \mathrm{~kb}$ (Chr13:5,109,848-5,114,847) promoter sequence of duck MITF gene was intercepted for bioinformatical analysis.

\section{Molecular evolution analysis}

The coding region sequence (CDS) of each duck POU gene family member was downloaded from NCBI. There was a total of 13 lines in the duck genome (IASCAAS_PekingDuck_PBH1.5, GCF_003850225.1). Evolutionary analyses were conducted in MEGA7[40]. The evolutionary relations were inferred using the Maximum Likelihood method based on the Hasegawa-Kishino-Yano model ${ }^{[41]}$. The bootstrap consensus tree inferred from 1000 replicates ${ }^{[42]}$ was taken to represent the evolutionary history of the taxa analyzed ${ }^{[42]}$. Branches corresponding to partitions reproduced in less than $50 \%$ bootstrap replicates are collapsed. 


\section{Data statistic}

One-way ANOVA analyzed all the data with the SPSS19.0 software package. Differences at $p<0.05$ were considered significant.

\section{Abbreviations}

GWAS

Genome-wide association studies

UV

ultraviolet rays

MITF

Melanocyte Inducing Transcription Factor

POU2F3

POU class 2 homeobox 3

MC1R

Melanocortin 1 Receptor

a-MSH

alpha-melanocyte-stimulating hormone

MAF

minor allele frequency

$\mathrm{AOI}$

the area of interest

MSA

melanin spot area in duck beak skin

BA

beak area

MSPBA

the melanin distribution per unit area of beak skin

CDS

coding region sequence

LD

Linkage disequilibrium

SNP

single-nucleotide polymorphism

\section{Declarations}

Ethics approval and consent to participate 
All methods were carried out in accordance with relevant guidelines and regulations. The protocols for all animal experiments were approved by the Animal Care and Use Committee of the Institute of Animal Sciences, Chinese Academy of Agricultural Science (CAAS), and all methods strictly obeyed the Guide for the ARRIVE (Animal Research: Reporting of In Vivo Experiments) guidelines 2.0 $0^{[43]}$.

\section{Consent for publication}

Not applicable.

\section{Competing interests}

Not applicable.

\section{Availability of data and materials}

The Genome sequences have been deposited in the Sequence Read Archive (https://www.ncbi.nlm.nih.gov/sra) with the accession codes PRJNA471401 and PRJNA450892.

\section{Funding}

This work was supported by grants from the National Natural Science Foundation of China (31972523 and 31872345), the National Scientific Supporting Projects of China (2015BAD03B06), the China Agriculture Research System of Waterfowl (CARS-42), and the CAAS Innovation Team Project (ASTIP2016-IAS-9, CAAS-XTCX2016010-03).

\section{Authors' contributions}

All experiments were designed by $\mathrm{HL}, \mathrm{ZZ}$ and $\mathrm{SH}$. LW, ZG, WF and $\mathrm{YX}$ performed the data analysis of genome. JH, DL, YZ, MX, JT and WH participated in the sample collection and preparation work. HL, JW, and QZ completed the manuscript writing. All authors have read and approved the manuscript.

\section{Acknowledgments}

Thanks to all the authors for their contributions to the study.

\section{References}

1. Glogau R.G. Physiologic and structural changes associated with aging skin. Dermatol Clin. 1997;15(4): p. 555-9.

2. RittiéL, G.J. Fisher. Natural and sun-induced aging of human skin. Cold Spring Harb Perspect Med. 2015; 5(1): p. a015370.

3. Chung J.H. Photoaging in Asians. Photodermatology, Photoimmunology \& Photomedicine. 2003; 19(3): p. 109-121. 
4. Peres P. Photoaging and chronological aging profile: Understanding oxidation of the skin. Journal of Photochemistry and Photobiology B: Biology. 2011; 103(2): p. 93-97.

5. Gilchrest B.A, F.B. Blog, G. Szabo. Effects of aging and chronic sun exposure on melanocytes in human skin. J Invest Dermatol. 1979; 73(2): p. 141-3.

6. HAWK, J.L.M. Photosensitivity in the elderly. British Journal of Dermatology. 1990; 122(s35): p. 2941.

7. Lin J.Y, D.E. Fisher. Melanocyte biology and skin pigmentation. Nature. 2007; 445(7130): p. 843-850.

8. Wihlmark U. Lipofuscin accumulation in cultured retinal pigment epithelial cells causes enhanced sensitivity to blue light irradiation. Free Radic Biol Med. 1997; 22(7): p. 1229-34.

9. Szabo G. The regional anatomy of the human integument with special reference to the distribution of hair follicles, sweat glands and melanocytes. Philosophical Transactions of the Royal Society of London. Series B. Biological Sciences, 1967; 252(779): p. 447-485.

10. Snell R.S, P.G. Bischitz. The melanocytes and melanin in human abdominal wall skin: asurvey made at diffenent ages in both sexes and during pregnancy. J Anat. 1963; 97(Pt 3): p. 361-76.

11. Ortonne J.P. Pigmentary changes of the ageing skin. Br J Dermatol. 1990; 122 Suppl 35: p. 21-8.

12. Costin G.E, V.J. Hearing. Human skin pigmentation: melanocytes modulate skin color in response to stress. Faseb j. 2007; 21(4): p. 976-94.

13. Tsatmali M, J. Ancans, A.J. Thody, Melanocyte function and its control by melanocortin peptides. J Histochem Cytochem. 2002; 50(2): p. 125-33.

14. Rouzaud F. MC1R and the response of melanocytes to ultraviolet radiation. Mutation Research/Fundamental and Molecular Mechanisms of Mutagenesis. 2005; 571(1): p. 133-152.

15. Thody A.J, A. Graham. Does alpha-MSH have a role in regulating skin pigmentation in humans? Pigment Cell Res. 1998; 11(5): p. 265-74.

16. Goodman G, D. Bercovich. Melanin directly converts light for vertebrate metabolic use: Heuristic thoughts on birds, Icarus and dark human skin. Medical hypotheses. 2008; 71: p. 190-202.

17. Zhou Z. An intercross population study reveals genes associated with body size and plumage color in ducks. Nature Communications. 2018; 9(1): p. 2648.

18. Lerner A.B. Metabolism of phenylalanine and tyrosine. Advances in Enzymology. 2009; 14: p. 73128.

19. Maddodi N,A. Jayanthy, V. Setaluri. Shining light on skin pigmentation: the darker and the brighter side of effects of UV radiation. Photochem Photobiol. 2012; 88(5): p. 1075-82.

20. Hitselberger M.H, R.L. Schleicher, C.W. Beattie. Effects of estradiol on estrogen receptor, progesterone receptor, and tyrosinase in hamster melanoma transplanted into athymic mice. Cancer Res. 1988; 48(13): p. 3720-7.

21. Jee S.H. Effects of estrogen and estrogen receptor in normal human melanocytes. Biochem Biophys Res Commun. 1994; 199(3): p. 1407-12. 
22. Kim N.H. PDZK1 upregulation in estrogen-related hyperpigmentation in melasma. J Invest Dermatol. 2012; 132(11): p. 2622-31.

23. Maranduca M.A. Synthesis and physiological implications of melanic pigments. Oncol Lett. 2019; 17(5): p. 4183-4187.

24. Scherer D, R. Kumar. Genetics of pigmentation in skin cancer-a review. Mutat Res. 2010; 705(2): p. 141-153.

25. Carreira S. Mitf regulation of Dia1 controls melanoma proliferation and invasiveness. Genes \& development. 2006; 20(24): p. 3426-3439.

26. Vachtenheim J J. Borovanský, "Transcription physiology" of pigment formation in melanocytes: Central role of MITF. Experimental dermatology. 2010; 19: p. 617-27.

27. Herr W. The POU domain: a large conserved region in the mammalian pit-1, oct-1, oct-2, and Caenorhabditis elegans unc-86 gene products. Genes Dev. 1988; 2(12a): p. 1513-6.

28. Besch R, C. Berking. POU transcription factors in melanocytes and melanoma. Eur J Cell Biol. 2014; 93(1-2): p. 55-60.

29. Wan P, Y. Hu and L. He. Regulation of melanocyte pivotal transcription factor MITF by some other transcription factors. Molecular and cellular biochemistry. 2011;354(1-2): p. 241-246.

30. Rawlings A.V. Ethnic skin types: are there differences in skin structure and function? Int J Cosmet Sci. 2006; 28(2): p. 79-93.

31. Brenner M. and V.J. Hearing. The protective role of melanin against UV damage in human skin. Photochemistry and photobiology. 2008; 84(3): p. 539-549.

32. Sturm R.A. Molecular genetics of human pigmentation diversity. Hum Mol Genet. 2009; 18(R1): p. R917.

33. Patel R.K. and M Jain. NGS QC Toolkit: A Toolkit for Quality Control of Next Generation Sequencing Data. PLOS ONE. 2012; 7(2): p. e30619.

34. Li H, R Durbin. Fast and accurate short read alignment with Burrows-Wheeler transform. Bioinformatics. 2009; 25(14): p. 1754-1760.

35. DePristo M.A. A framework for variation discovery and genotyping using next-generation DNA sequencing data. Nature Genetics. 2011; 43(5): p. 491-498.

36. Danecek P. The variant call format and VCFtools. Bioinformatics, 2011; 27(15): p. 2156-8.

37. Kang H.M. Variance component model to account for sample structure in genome-wide association studies. Nature Genetics. 2010; 42(4): p. 348-354.

38. Price A.L. Principal components analysis corrects for stratification in genome-wide association studies. Nature genetics. 2006; 38(8): p. 904-909.

39. Eisen M.B. Cluster analysis and display of genome-wide expression patterns. Proceedings of the National Academy of Sciences. 1998; 95(25): p. 14863-14868.

40. Kumar S. G, Stecher, K. Tamura. MEGA7: molecular evolutionary genetics analysis version 7.0 for bigger datasets. Molecular biology and evolution. 2016;33(7): p. 1870-1874. 
41. Hasegawa M, H. Kishino, T.-a. Yano. Dating of the human-ape splitting by a molecular clock of mitochondrial DNA. Journal of Molecular Evolution. 1985; 22(2): p. 160-174.

42. Felsenstein J. confidence limits on phylogenies: an approach using the bootstrap. Evolution. 1985; 39(4): p. 783-791.

43. Percie du Sert. Reporting animal research: Explanation and elaboration for the ARRIVE guidelines 2.0. PLOS Biology. 2020; 18(7): p. e3000411.

\section{Figures}


A

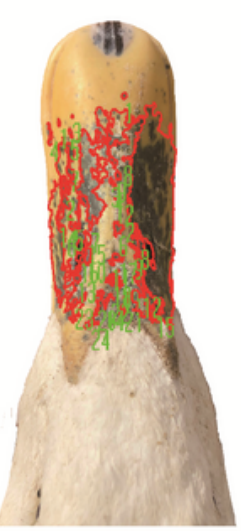

C

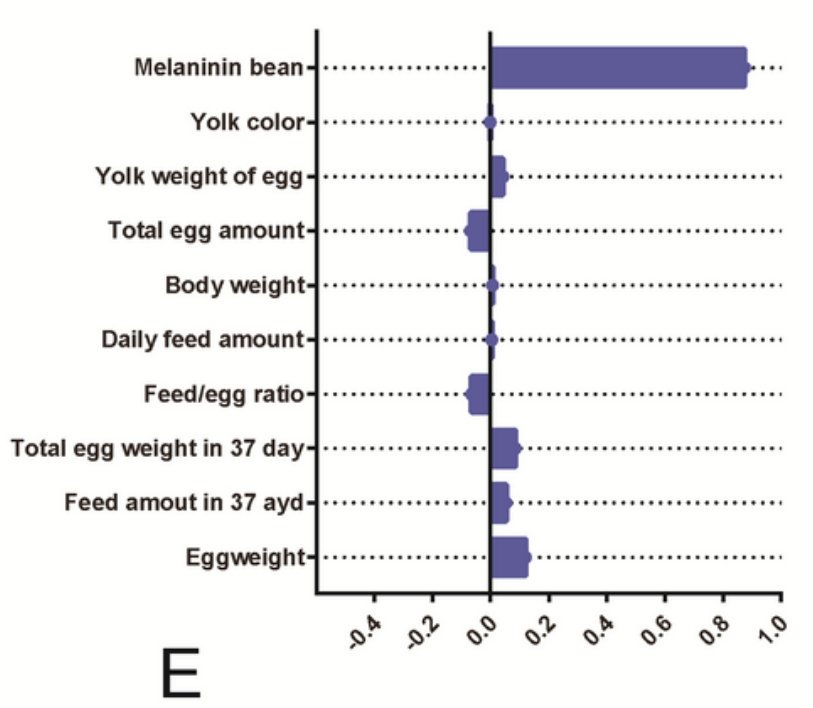

B

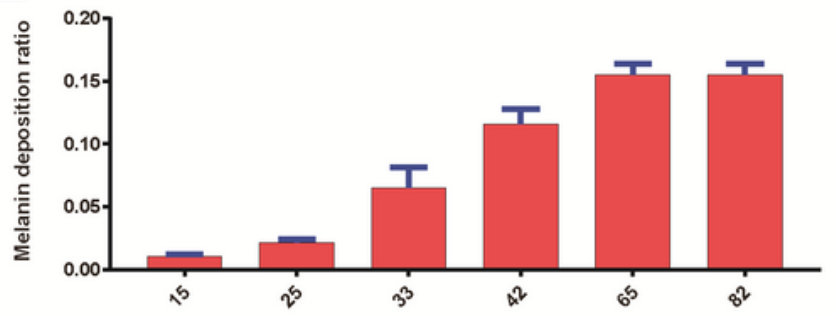

D

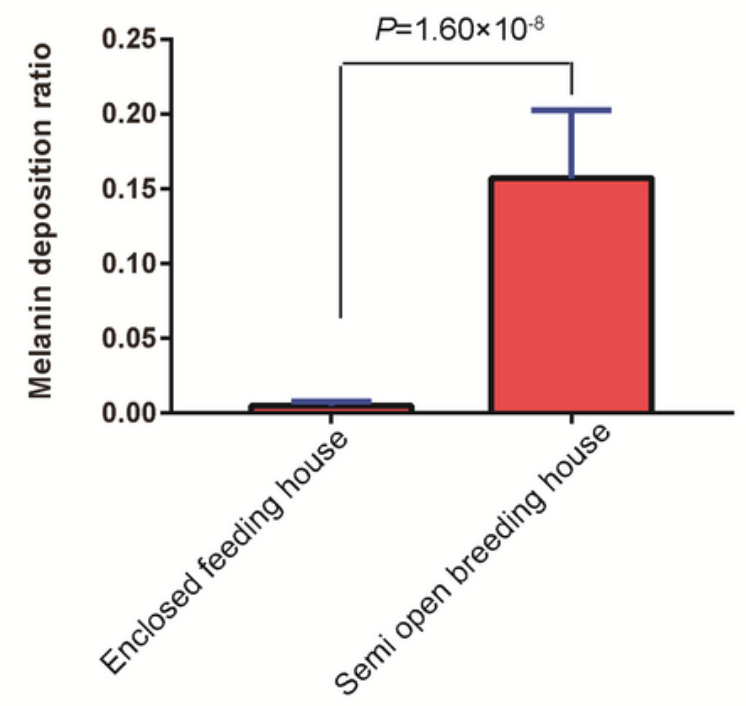

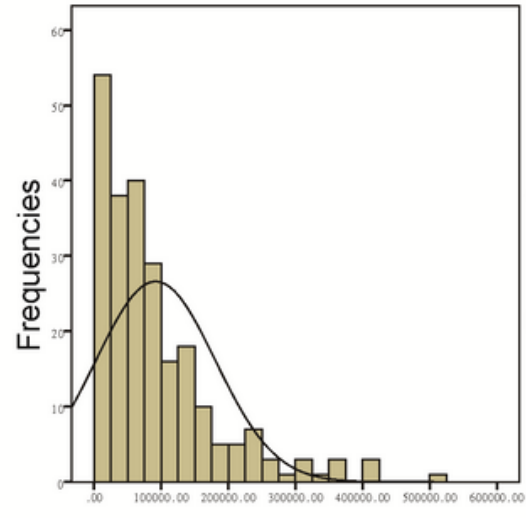

MSA

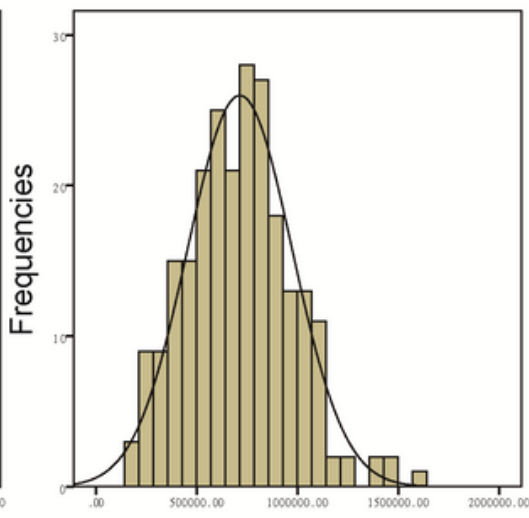

BA

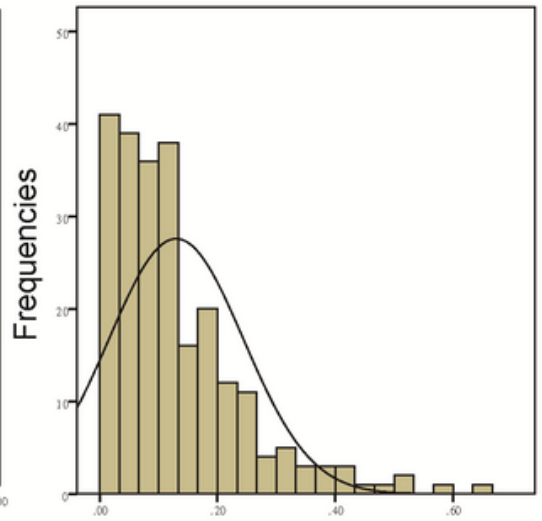

MSPBA

\section{Figure 1}

Phenotypic observations of melanin spot in duck beak skin. A: IPP 6.0 was used for determining the melanin spot amount (MSA), beak area (BA), and the melanin spot amount per unit beak area (MSPBA). The red dot marked on the left beak showed the melanin spot distribution by the irregular function of IPP 6.0. The red line marked the beak on the right side is the region for calculating the beak area. $\mathrm{B}$ : The melanin spot was accumulated accompanying the age of ducks. C: The phenotypic correlations between 
MSPBA with reproduction-related traits. D: The MSPBA was compared between ducks under an enclosed house (with no sunlight exposure during the growing period) and a semi-open breeding house (exposed to sunlight during the growing period). E: The distribution states of phenotypic values including MSA, BA, and MSPBA from 223 ducks.
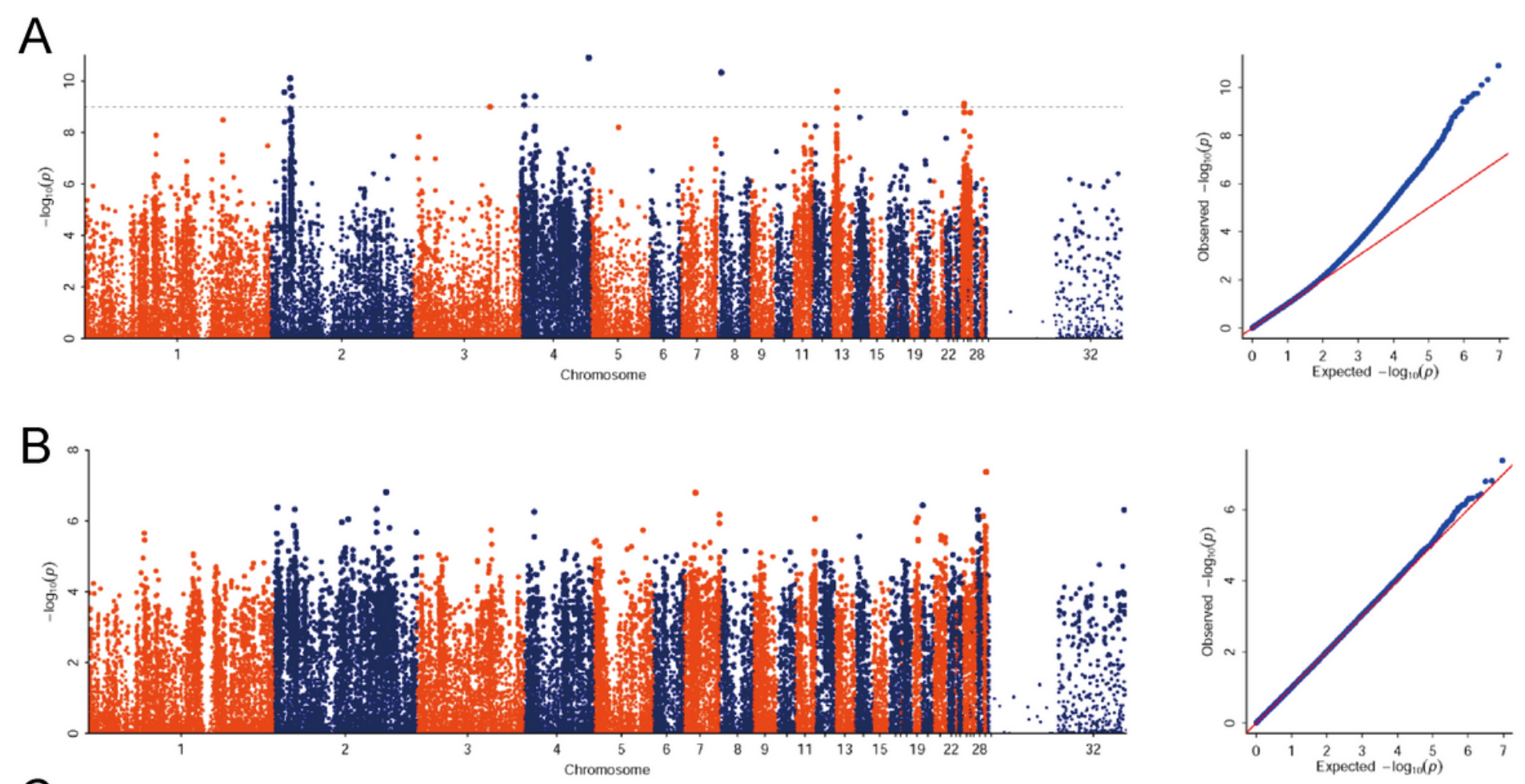

C
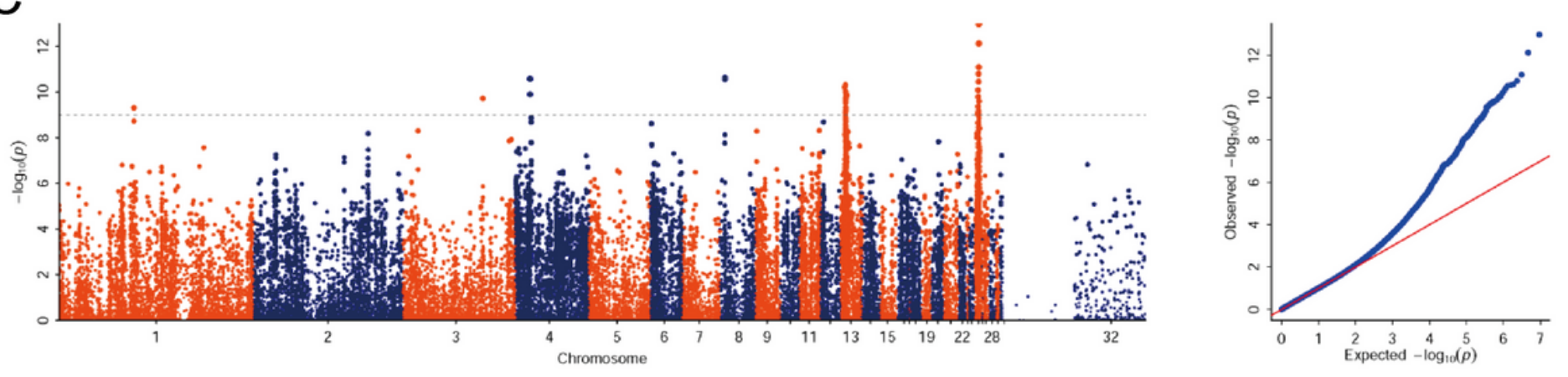

Figure 2

Manhattan (left) and QQ plots (right) showing the significance of genetic effects on the Melanin spot in duck beak skin by a GWAS. A: For melanin spot amount (MSA); B: For beak area (BA); and C: For melanin spot amount per unit beak area (MSPBA). The GWAS was performed in 223 F2 ducks crossed by Pekin ducks and mallards. The gray line represents the Bonferroni corrected significance threshold $(-\log 10 \mathrm{P}=$ 8.16). The $x$-axis shows the physical positions for each marker along the chromosomes, and the $y$-axis shows the - $\log 10 \mathrm{P}$ values for the association tests. 
A
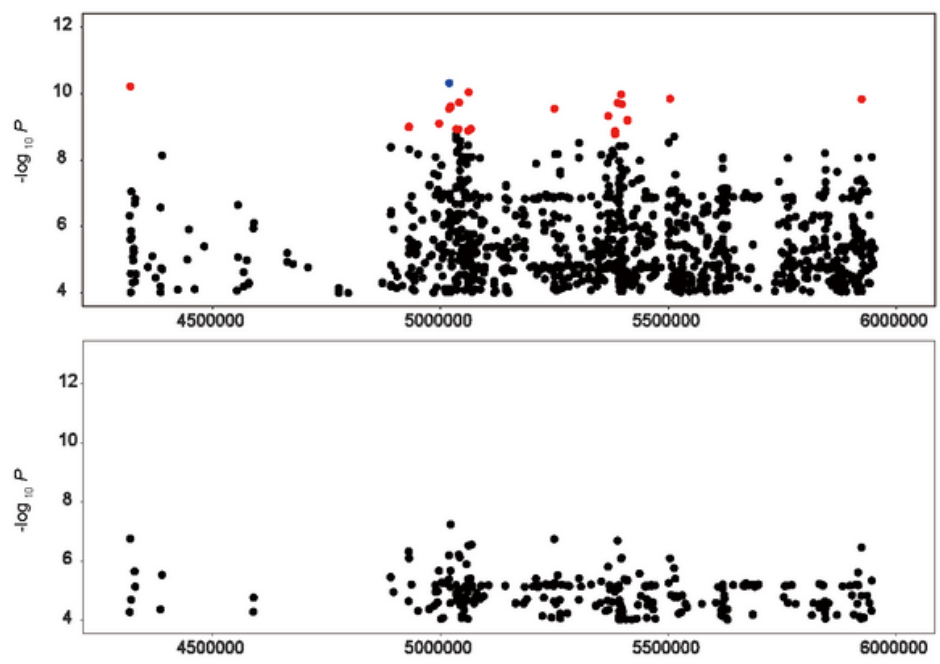

C

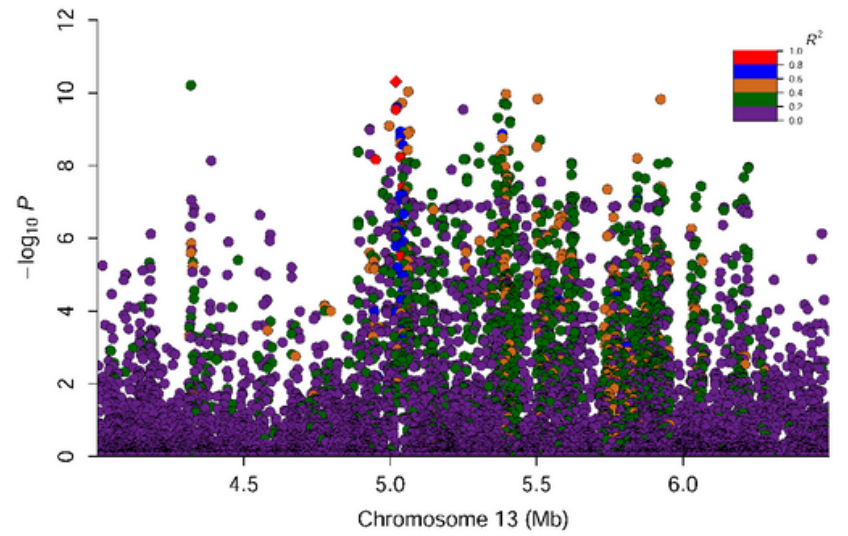

B
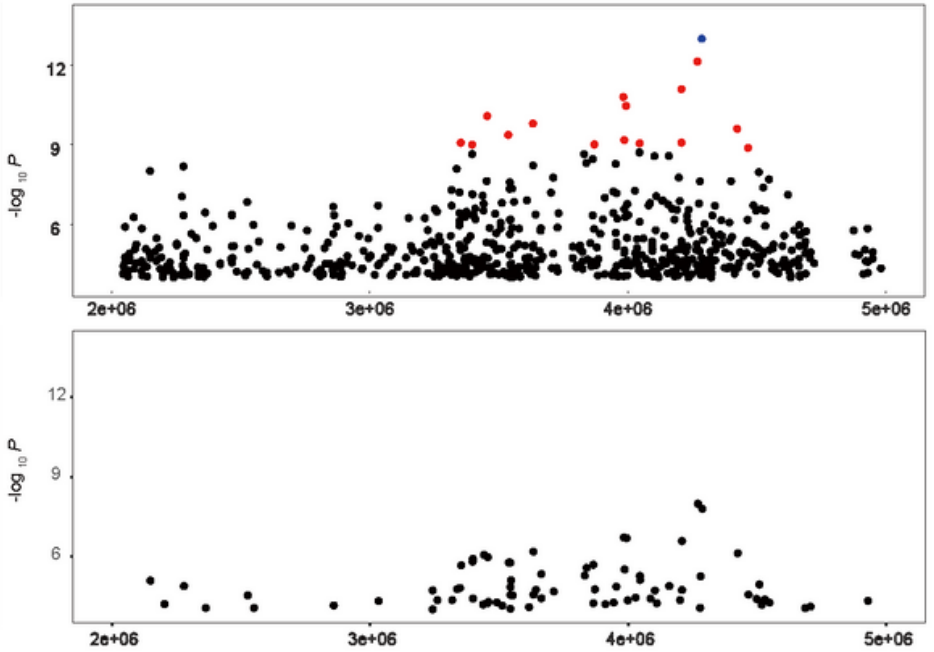

D

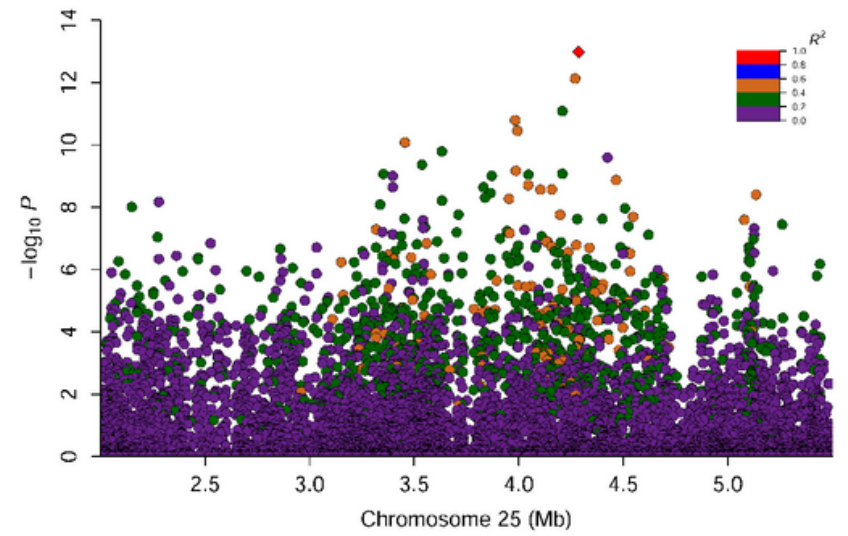

Figure 3

Conditional GWAS and regional plots of the loci associated with MSPBA. A \& B. Conditional association analyses were carried out by fitting the most significant SNP Chr13:5,019,135 ( $P=4.88 \times 10-11)$ and Chr25:42,867,656 ( $\mathrm{P}=1.04 \times 10-13)$ as a covariate for the melanin spot amount per unit beak area (MSPBA), respectively. C\&D. Regional plots for the loci associated with MSPBA. All genotyped SNPs are color-coded according to their pairwise LD with the sentinel SNP (Chr13:5,019,135 and Chr25:42,867,656) calculated in the F2 intercross population. The strong LD block is defined as R2 $\geq 0.4$. 
A

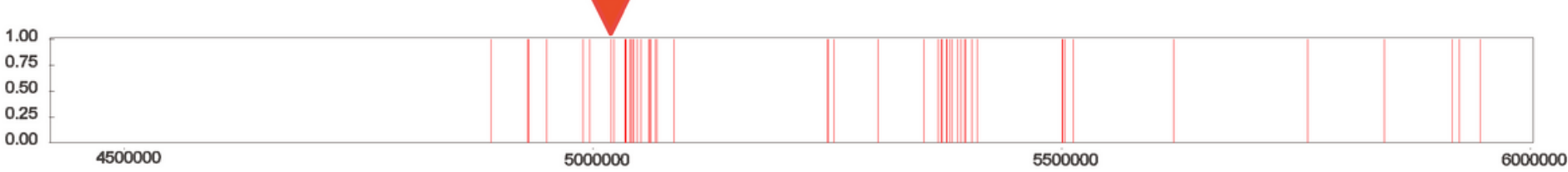

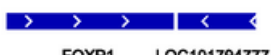

FOXP1_LOC101794777 P-IIIH

-

XM 027467452.1

Ю 1 HНHн—

XM_021266696.2

$\stackrel{\longrightarrow}{\longrightarrow}$ IIIH冊

XM_021266699.2



XM_013091805.3

Ю 1 W1

XM 013091809.3

F十川11曲

XM 027467453.1

I 1 H

XM_027467454.1

XM 0274674551

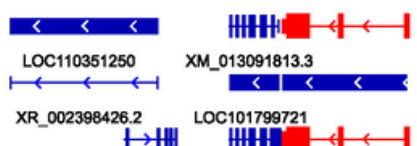

XR_002398426.2 LOI LOC10179972

XM_027467748.1

MDFIC2 LOC101799904

P, III

XM_021266702.2
XM_027467277.1

XM_013091817.3

XM_ 013091817

XM_021266705.

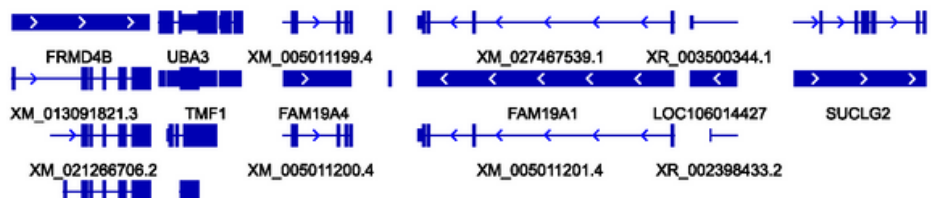

XM_021266706.2

HIIII

MM_027467444.1

XM_027467445.

HHH

XM 027467446

HHH

XM_013091823.3

XM_013091819.3

B

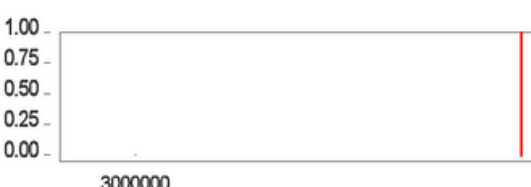

\& > < > $<1$

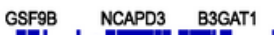

내 H) if :

7444127.1 XM_027444128.1

LOC106014459

HH

XR_003492464.1

IIIH H

XR_003492463.1

|

XR_003492460.1

it

XR_003492461.1

배

XR_003492462.1

H-

H-

XM_027444263.1

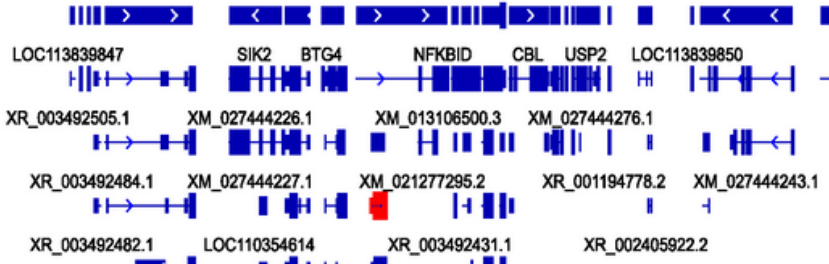

2.1 LOC110354614

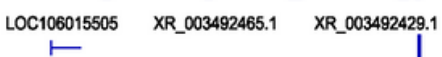

XR_001187589.3

XM_005027095.4

XR_001187587.3

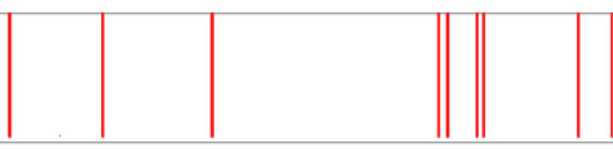

4000000

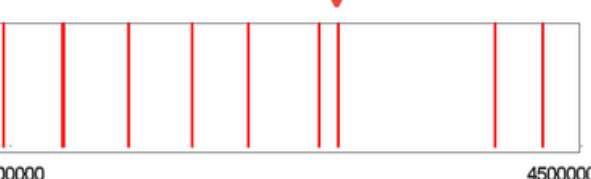

LOC110353386

XR_002403818.2

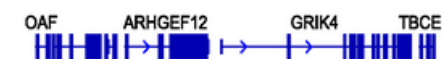

XM_013103115.3 XM_027444301.1

a I H H $\rightarrow$

TRIM29 XM 013103114.3

IIH IIIH

XM_027444322.1

XM_027444143.1

LOC113839833

H.

XR_003492439.1

H

XM_027444215.1

4

XM_027444213.1

\section{Figure 4}

The distributions of significant SNPs and genes in the candidate chromosomal region. A: The candidate genes in Chr13. B: The candidate genes in Chr25. The reference duck genome was IASCAAS_Peking Duck_PBH1.5 (GCF_003850225.1), a top-level updated duck genome with N50 = 76,129,154, downloaded from NCBI. The triangle fulfilled in red represents the locations of the leader significant SNPs. Each vertical red line represents an SNP associated with melanin spot deposition that reached the significant threshold line of $-\log 10 P=8.16$. The gene names in red were the potential candidate genes according to the expression profiles among tissues, supported by transcriptome from a global duck expression dataset. 
A
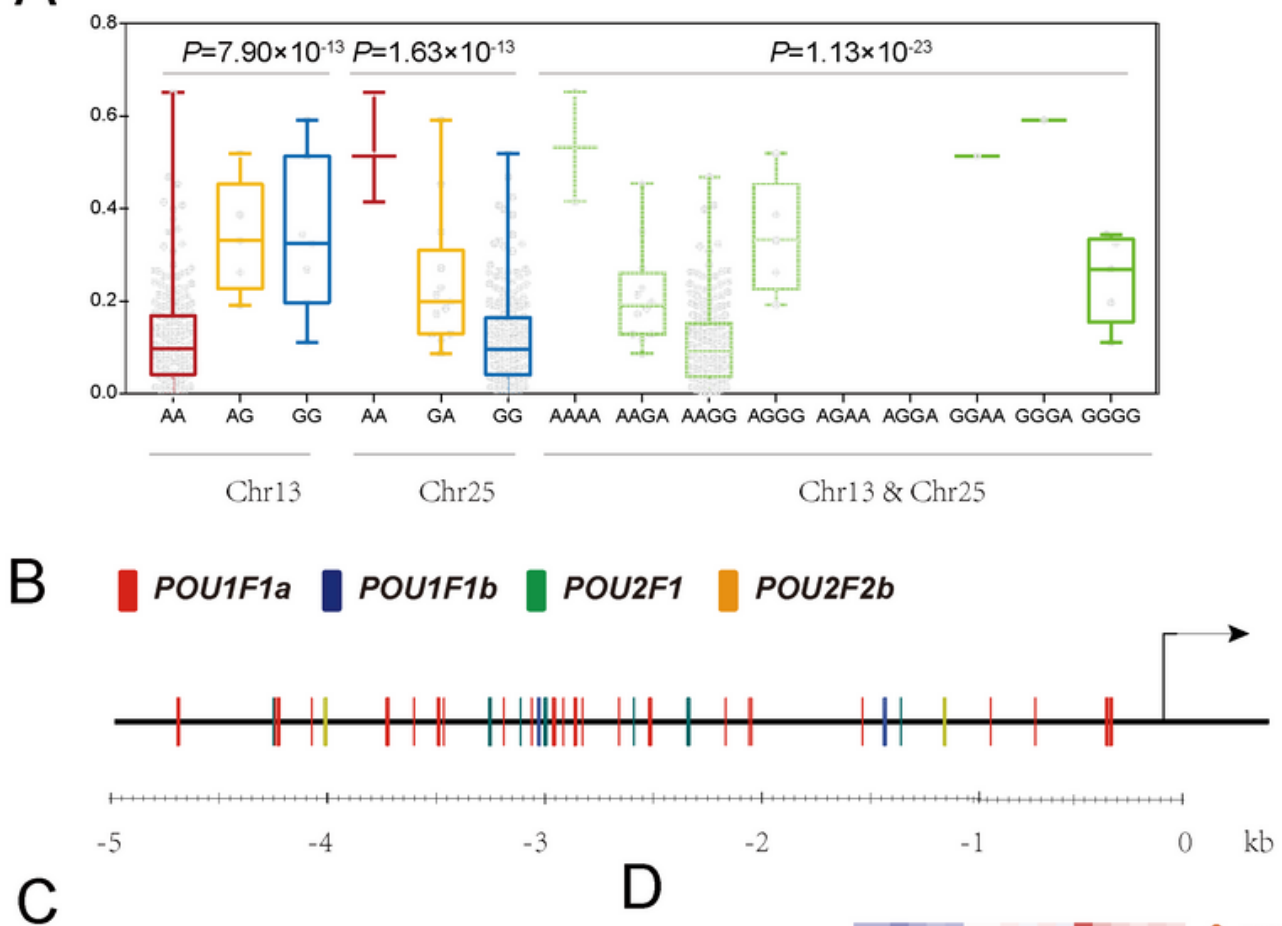

C

D
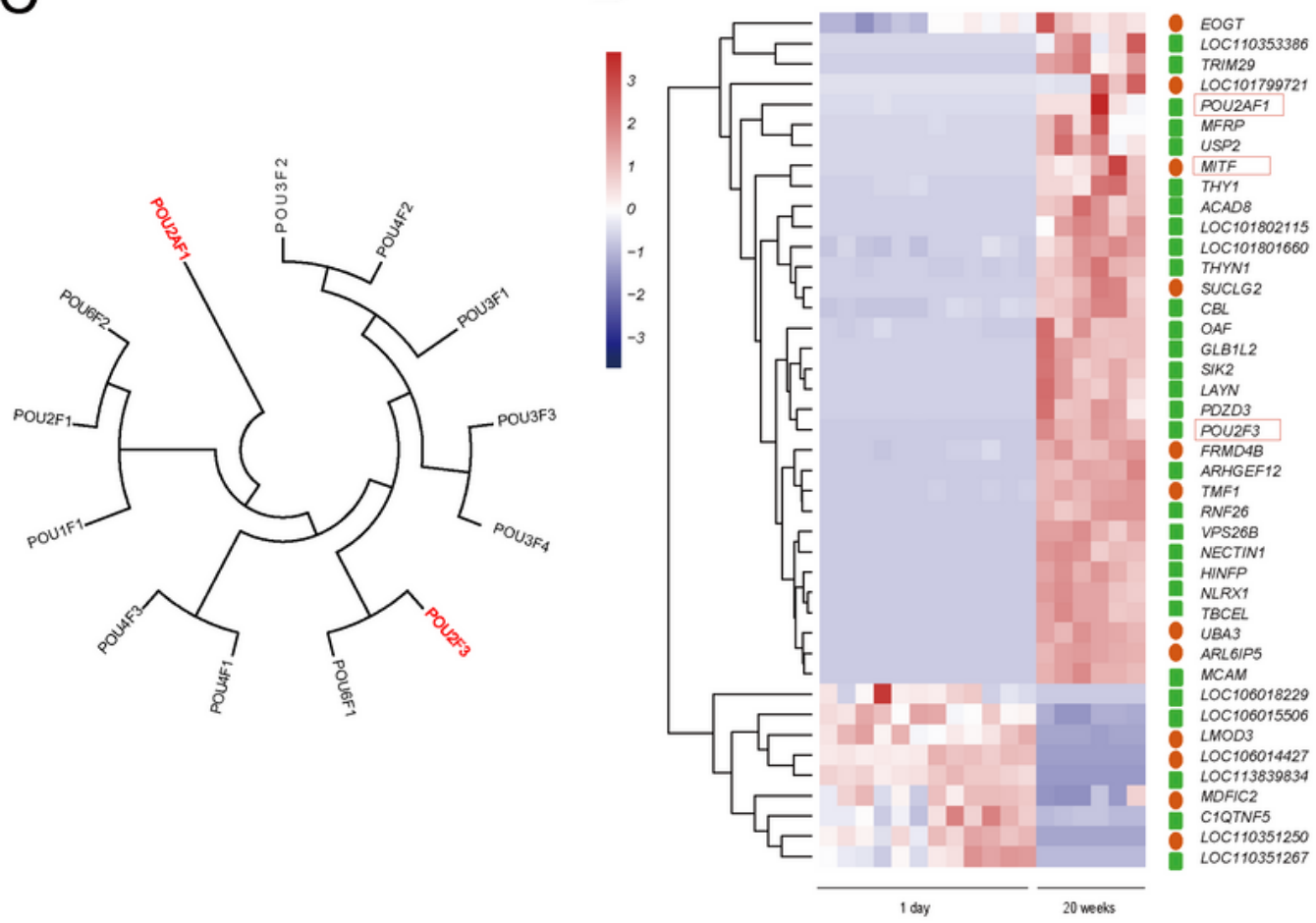

Figure 5

The potential regulatory relationships existed between MITF and POU transcription factors. A: Box plot for effect of sentinel SNPs on Chr13 and Chr25 (Chr13:5,019,135 and Chr25:42,867,656) on MSPBA in 223 ducks. The indicated $P$ values are based on one-way ANOVA. Box plots denote median (centerline), 2575th percentile (limits), minimum and maximum values (whiskers). B: the distribution of genomic locations of POU gene family transcription factors predicted within the cis-regulatory region, the sequence 
of $5 \mathrm{~kb}(\mathrm{Chr} 13: 5,109,848-5,114,847)$ ahead of the transcription site of the MITF gene in duck genome. All POU transcription factors were provided in Table S3. C: the molecular phylogenetic relationships of duck POU gene family members based on the Maximum Likelihood method. The gene members marked in red were predicted as the potential transcription factors of the duck MITF gene. D: the cluster analysis of gene expression profiles of candidate genes. The circles fulfilled in red ahead of the gene names represent the genes located in Chr13, while the square fulfilled in green represents the genes located in Chr25.

\section{Supplementary Files}

This is a list of supplementary files associated with this preprint. Click to download.

- TableS2.xIsx

- TableS1.xIsx

- TableS3.xIsx

- Figures1.tif

- FigureS2.tif

- SupplementaryFileLegends.docx 